Artículo

Los textos publicados son responsabilidad exclusiva de sus autores

\title{
Utilización de los conectores discursivos en la construcción textual de contenido axiológico
}

\section{Use of discursive connectors in the textual Construction of axiological content}

\author{
Yarisleidy Castellón Perez \\ Maestría en Dirección del Proceso Educativo \\ Universidad de Ciego de Ávila: Máximo Gómez Báez, Cuba \\ yarilester2018@gmail.com \\ https://orcid.org/0000-0001-6229-2908
Israel León Martínez
Universidad de Ciego de Ávila: Máximo Gómez Báez, Cuba
israelleonmartinez@gmail.com \\ Kenia González González \\ Universidad de Ciego de Ávila: Máximo Gómez Báez, Cuba \\ ksolenzal@gmail.com
}

\section{Referencia}

Castellón Perez, Y., León Martínez, I. y González González, K. (2021). Utilización de los conectores discursivos en la construcción textual de contenido axiológico. Revista Guatemalteca de Educación Superior, 4(1), 23-31. DOI: https://doi.org/10.46954/revistages.v4i1.51

Recibido: 15/11/2020

Aceptado: 16/01/2021

\section{Resumen}

En este artículo se enfatizó en la utilización de los conectores discursivos en la construcción de textos escritos de contenido axiológico con un marcado carácter reflexivo teniendo en cuenta para ello el pensamiento de Ernesto Guevara y Fidel Castro y su vigencia en la sociedad actual, se seleccionaron estudiantes de la Educación Preuniversitaria para la población y muestra que presentaban dificultades en la utilización de los conectores del discurso, se constató a partir de un diagnóstico inicial la problemática, se elaboró y aplicó un sistema de 
ejercicios y posteriormente se implementó una comprobación final para constatar la eficacia del mismo, se obtuvo que se fortaleció la formación de valores y el modo de actuación de las futuras generaciones de cubanos de forma tal que perduren

Palabras clave:

Contenido axiológico, conectores discursivos, construcción textual. las conquistas alcanzadas por la revolución y se perfeccionó la utilización de los conectores discursivos en la construcción de textos escritos.

\section{Keywords:}

Axiological content, discursive connectors, textual construction.

\section{Abstract}

This article emphasizes the use of discursive connectors in the Construction of written texts with axiological content with a marked reflective character taking into account the thought of Ernesto Guevara and Fidel Castro and its validity in today's society, students of pre-university education were selected for the population and shows that they had difficulties in the use of discourse connectors, it was verified from an initial diagnosis the problem, a system of exercises and exercises was developed and applied subsequently a final check was implemented to verify its effectiveness, it was obtained that the formation of values and the mode of action of future generations of cubans were strengthened in such a way that the conquests achieved by the revolution would last and the use of discursive connectors was perfected in the construction of written texts. Introducción

\section{Introducción}

El dominio de la lengua materna constituye una necesidad en la sociedad, pues esta participa en los procesos de cognición, comunicación y expresión humanas. Sobre la base de esta premisa, la sociedad cubana se proyecta mediante el sistema educativo con el fin de optimizar el proceso de enseñanzaaprendizaje de la asignatura Español-Literatura en los diferentes niveles educativos.

A partir de la intencionalidad, antes esgrimida, en el contexto educacional de la Educación Preuniversitaria se cumple el Programa Director de la Lengua Materna orientado por el 
Ministerio de Educación (2020), a favor de que los estudiantes desarrollen las cuatro macrohabilidades generales de la lengua materna: escuchar, hablar, leer y escribir, pues la concepción actual de la enseñanza de la lengua se basa en un enfoque cognitivo-comunicativo y sociocultural propiciando la competencia comunicativa de los estudiantes.

Sin embargo, la realidad revela que pese al carácter integrador que debe tener el proceso de enseñanza-aprendizaje de la asignatura Español-Literatura en la Educación Preuniversitaria persisten formas y métodos tradicionales que no estimulan en los estudiantes, de manera suficiente, en el empleo de los conectores discursivos para la construcción de textos escritos ya sean literarios y no literarios.

Al respecto los autores se adhieren favorablemente al criterio de José Alberto López, quien considera que los conectores o marcadores del discurso son: unidades lingüísticas invariables (conjunciones, preposiciones, adverbios, frases preposicionales, locuciones adverbiales) que no ejercen una función sintáctica en el marco de la predicación oracional, sirven para dar estructura al texto, es decir, para establecer orden y relaciones significativas entre sus partes y para estructurar las ideas dentro de él y conectarlas entre sí en el interior de la oración (López, J, 2017, p. 84).

Los conectores discursivos, ocupan un lugar esencial dentro del enfoque cognitivo-comunicativo que persigue la asignatura Español-Literatura en el preuniversitario y su empleo con eficacia contribuye a que el estudiante perciba el léxico no como una simple suma de palabras sino como un todo sistémico; para así estar en mejores condiciones de interpretar y construir eficientemente el texto escrito, no solo en la asignatura antes mencionada sino en todas de forma general.

La utilización de los conectores discursivos para la construcción de los textos escritos tiene una influencia importante en el desarrollo de la personalidad de los estudiantes de preuniversitario debido a que estos jóvenes, están potencialmente aptos para realizar 
tareas que requieren una alta dosis de trabajo mental, de razonamiento, iniciativa, inventiva, imaginación y creatividad. Por tanto, la utilización de los conectores del discurso, posibilita atribuirle al texto sentidos y significaciones para su crecimiento personal.

La educación desde la instrucción es un principio de la pedagogía cubana, es por ello que para el logro de la formación integral de la personalidad del estudiante, se fusionan en este material las asignaturas Español Literatura e Historia de Cuba con el objetivo de fortalecer el proceso de enseñanza aprendizaje de la construcción del texto escrito mediante la utilización de los conectores del discurso a partir del reconocimiento, clasificación y uso de los mismos en textos expositivos y argumentativos que fortalezcan los valores de responsabilidad, laboriosidad, patriotismo, honestidad entre otros más que deben caracterizar a todo ser humano en cada una de las sociedades donde viva y se desenvuelva.

Para darle cumplimiento a lo anteriormente expuesto se elaboraron sistemas de ejercicios utilizando textos que evidencian el pensamiento económico, político y social de dos personalidades de nuestra historia: Ernesto Guevara y Fidel castro Ruz con el objetivo de que los estudiantes una vez leído los textos, los comprendan y expresen a través de textos escritos expresen sus criterios al respecto en los que se aprecien el uso correcto de los conectores del discurso.

A continuación, ofrecemos una muestra del sistema de ejercicios aplicado:

\section{Primer sistema de ejercicios}

Lee detenidamente el siguiente texto y luego realiza las actividades propuestas.

El Che en sus análisis acerca de la Ley del valor, consideró que, a diferencia del capitalismo, en la construcción del socialismo 
es un proceso conscientemente, deliberado, volitivo, dirigido a superar las contradicciones y desigualdades para crear una nueva forma de vida humana. La distribución del trabajo y la riqueza, por tanto, tiene que ser también deliberadamente, de forma planificada. La planificación es la vía fundamental, no la única, pero sí la fundamental para que los hombres dirijan la distribución justa de las riquezas.

a) El texto anterior es: __iterario o _ _ no literario.

b) Extrae el primer sintagma nominal, escriba su estructura gramatical.

c) Identifique y extraiga del texto cinco conectores del discurso. Clasifíquelos atendiendo a su categoría gramatical.

d) Construye un párrafo en el que argumentes la última oración psicológica del texto, no olvides hacer un adecuado empleo de los conectores discursivos.

\section{Segundo sistema de ejercicios}

Lea detenidamente el siguiente texto y relacionado con él realice las actividades propuestas:

Ernesto Guevara y Fidel Castro son paradigmas del ejemplo a seguir en la construcción del socialismo como ciencia del ejemplo. El pensamiento económico de ambos, es síntesis del estudio profundo de las aportaciones del Marxismo Leninismo, conjugado armónicamente con la interpretación de la realidad económica, política y social del mundo, así como de las mejores experiencias en la construcción de una nueva sociedad, capaz de dar respuestas a los males del capitalismo en su bregar histórico por plagar de miseria y desigualdad a los seres humanos, en interés de la acumulación de capital a favor de unos pocos en detrimento de muchos. 
a) Resuma la idea esencial del texto anterior.

b) El texto inicial tiene carácter:

c) Identifique y extraiga cinco conectores del discurso y clasifíquelos atendiendo a su categoría gramatical.

d) ¿Por qué podemos afirmar que el Che y Fidel son paradigmas a seguir en la construcción del socialismo? Ejemplifique a través de la construcción del texto escrito su respuesta, no olvide hacer un adecuado uso de los conectores del discurso.

Estos sistemas de ejercicios pueden acompañarse para la motivación de las clases con fragmentos de videos seleccionados que guarden relación con el tema objeto de estudio y se pueden enriquecer también con más actividades. Materiales y métodos

En la investigación se utilizaron los métodos del nivel teórico: el histórico lógico, la modelación, el sistémico, el analíticosintético y el inductivo- deductivo, del empírico: la observación y el experimento pedagógico en su variante pre-experimental, auxiliados por entrevistas, encuestas, el análisis documental y el criterio de expertos; de la estadística descriptiva se utilizó el procedimiento análisis porcentual para la valoración de los resultados de los instrumentos aplicados.

\section{Resultados}

Con la implementación de esta tipología de ejercicios en clases de consolidación de conocimientos, no solo se elevó la calidad del aprendizaje en cuanto al reconocimiento, clasificación y aplicación de los conectores del discurso en la construcción de textos escritos, sino que; se formaron valores como el patriotismo, la solidaridad, el respeto y aprecio a los mártires caídos y a los logros de la revolución cubana en los educandos. 
El grado seleccionado para la aplicación de la propuesta fue el duodécimo, en la Educación Preuniversitaria, se tomó una población de cincuenta estudiantes y la muestra coincide de forma intencional con dicha población debido a las características del grado relacionadas con el tema objeto de estudio.

En un momento inicial con la aplicación de un primer diagnóstico relacionado con el reconocimiento, clasificación y aplicación de los conectores del discurso en las construcciones textuales se obtuvo que de los 50 estudiantes, 18 que representa el 36\%, sabían solo reconocer algunos conectores del discurso, 15 del total que representan el 30\%,sabían reconocerlos y clasificarlos atendiendo a la categoría gramatical a la cual pertenecían y 17 del total que representa el 34\%, los reconocían, clasificaban y aplicaban adecuadamente en los textos construidos. Luego de poner en práctica esta tipología de ejercicios se aplicó un segundo diagnóstico comprobatorio del cual se obtuvo que de los 50 estudiantes, 44 de ellos que representa el $88 \%$, los reconocían y clasificaban adecuadamente y solamente 6 del total que representaban el $12 \%$, quedaban con dificultad en cuanto a la utilización de los mismos. Con ellos se hizo atenciones diferenciadas enfatizando en la erradicación de la dificultad.

A continuación, lo mostramos gráficamente los diagnósticos inicial y final:

Tabla 1. Utilización de los conectores del discurso en la construcción del texto escrito.

\begin{tabular}{|l|l|l|l|l|l|l|}
\hline \multirow{2}{*}{$\begin{array}{l}\text { Población } \\
\text { y muestra. }\end{array}$} & \multicolumn{2}{|l|}{ Diagnóstico inicial } & \multicolumn{2}{l|}{ Diagnóstico final } \\
\cline { 2 - 7 } & $\begin{array}{l}\text { I Nivel } \\
\text { Reconocimiento }\end{array}$ & $\begin{array}{l}\text { II Nivel } \\
\text { Clasificación }\end{array}$ & $\begin{array}{l}\text { III Nivel } \\
\text { Utilización }\end{array}$ & $\begin{array}{l}\text { I Nivel } \\
\text { Reconocimiento }\end{array}$ & $\begin{array}{l}\text { II Nivel } \\
\text { Clasificación }\end{array}$ & $\begin{array}{l}\text { III Nivel } \\
\text { Utilización }\end{array}$ \\
\hline $50-100 \%$ & $18-36 \%$ & $15-30 \%$ & $17-34 \%$ & - & $44-88 \%$ & $6-12 \%$ \\
\hline
\end{tabular}




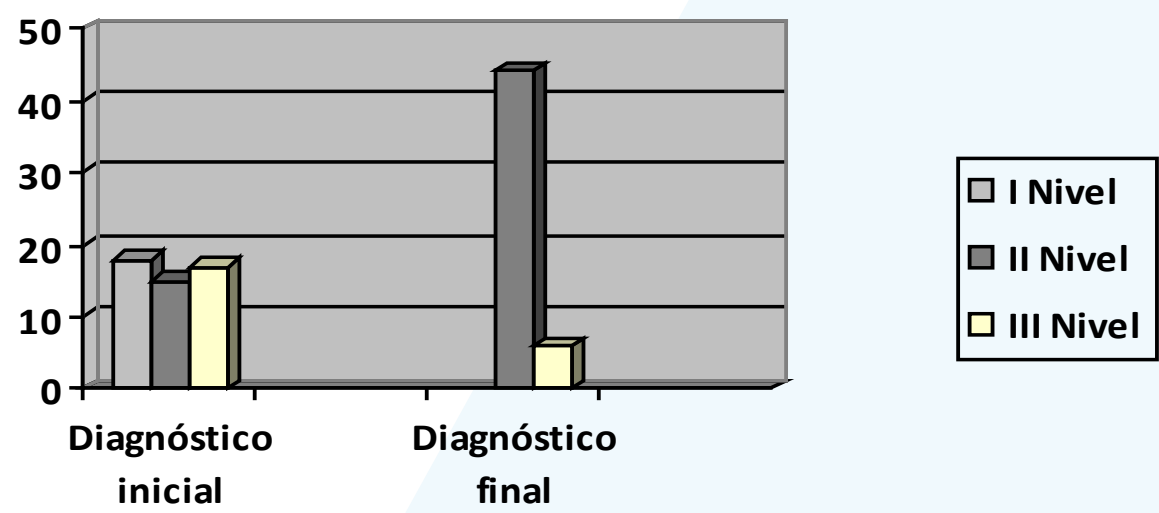

\section{Discusión}

Como se ilustra en el gráfico anterior hubo transformaciones positivas en cuanto a la aprehensión de los conocimientos de la utilización de los conectores discursivos en la construcción de textos escritos y con ello también se obtuvo la elevación de la calidad del trabajo político e ideológico en los educandos, contribuyendo así a su visibilidad con respecto a la sociedad en la cual ellos se desarrollan y con la cual deben contribuir en agradecimiento una vez terminados los estudios universitarios.

\section{Agradecimientos}

A los estudiantes de duodécimo grado del Instituto Preuniversitario Urbano: José Martí Pérez, del poblado Gaspar, municipio Baraguá de la provincia Ciego de Ávila por ser parte del estudio científico, a mi amiga Zuleiny Meneses Martín, máster en Ciencias de la Educación y licenciada en Español-Literatura, al Ministerio de Educación y a los profesores de la plataforma de Posgrado de la Universidad de Ciego de Ávila: Máximo Gómez Báez, por contribuir a mi formación durante este hermoso transitar por la maestría en Dirección del Proceso Docente Educativo III Edición y en especial a mis tutores y profesores titulares Dr.C Israel León Martínez y Dr.C Kenia González González. 


\section{Referencias}

Castellón Pérez, Y., León Martínez, I. y González González, K. (2021). Ejercicios para el empleo de los conectores discursivos en la construcción del texto escrito. RIPIE. 1(1), 2745-0341. https://doi.org/10.51660/ripie.v1i1

Castellón Pérez, Y., Meneses Martín, Z. y León Martínez I. (2020). Ejercicios para el uso de los conectores o marcadores discursivos. España, eae.

López Díaz, J. A. (2018). Compendio de gramática española y apuntes sobre redacción. La Habana, Cuba: Pueblo y Educación. pp. 84-85

Martín Pérez, O., Meneses Martín, Z., y Castellón Pérez, Y. (2020). Sistema de ejercicios para potenciar el conocimiento de la Historia. España, eae.

\section{Sobre los autores}

Investigadores de la Universidad de Ciego de Ávila, Cuba: Máximo Gómez Báez.

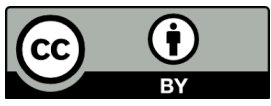

Este texto está protegido por una licencia CreativeCommons 4.0.

Usted es libre para compartir, copiary redistribuir el material en cualquier medio o formato y adaptar el documento, remezclar, transformar y crear a partir del material para cualquier propósito, incluso comercialmente, siempre que cumpla la condición de atribución: usted debe reconocer el crédito de una obra de manera adecuada, proporcionar un enlace a la licencia, e indicar si se han realizado cambios. Puede hacerlo en cualquier forma razonable, pero no de forma tal que sugiera que tiene el apoyo del licenciante o lo recibe por el uso que hace. 